\title{
LOS OBJETOS BIOTECNOLÓGICOS: CONCEPCIONES FILOSÓFICAS Y CONSECUENCIAS PARASU EVALUACIÓN
}

\section{León Olivé*}

\begin{abstract}
Resumen: Por su propia naturaleza, los sistemas biotecnológicos generan tanto beneficios como riesgos y situaciones de incertidumbre y de ignorancia. Las evaluaciones de los sistemas biotecnológicos, de los artefactos que producen y de sus consecuencias en las sociedades y en la naturaleza, dependen tanto de valores constitutivos de esos sistemas biotecnológicos, como de diferentes valores externos a ellos, que varían de un grupo social a otro y que muy difícilmente llegan a coincidir completamente. Tal diversidad valorativa debe ser reconocida en los procesos de evaluación de la biotecnología, por ejemplo sobre cuestiones de bioseguridad, por parte de expertos y de diversos sectores sociales (agentes estatales, empresarios, organizaciones no gubernamentales, grupos ciudadanos). En consecuencia, deben establecerse instancias de participación de expertos de diferentes disciplinas naturales, sociales y humanísticas, así como de no expertos, representantes de los sectores sociales con intereses en juego, que vigilen el impacto de los sistemas biotecnológicos y de sus artefactos y que tengan capacidad de tomar decisiones y de realizar acciones para prevenir, paliar o contrarrestar efectos negativos de los sistemas biotecnológicos.
\end{abstract}

Palabras clave: Biotecnología, ética y biotecnología, naturaleza de los objetos biotecnológicos, sistemas biotecnológicos, artefactos biotecnológicos

\section{BIOTECHNOLOGICAL OBJECTS: PHILOSOPHICAL CONCEPTIONS AND CONSEQUENCES FOR THEIR EVALUATION}

\begin{abstract}
Due to their intrinsic nature, biotechnological systems produce both benefits as well as risks and situations of uncertainty and ignorance. The evaluations of biotechnological systems, of the artifacts produced by them and of their consequences for society and the environment, depend both on intrinsic values of those systems as well as on values extrinsic to them, which in turn vary from one social group to another and which can hardly coincide completely. Such axiological plurality must be taken into account in reviewing biotechnology, for example about biosafety issues by experts from different social sectors (state agents, managers, non governmental organizations, advocacy groups) Therefore, mechanisms of participation of experts from different natural, social and humanistic disciplines must be established, along with non experts representing the social sectors with interests. These will watch over the impact of biotechnological systems and their artifacts, they will be able to take decisions and carry out actions to prevent, mitigate or counteract the negative consequences of biotechnology.
\end{abstract}

Key words: Biotechnology, biotechnology and ethics, nature of biotechnological objects, biotechnological systems, biotechnological artifacts

\section{LOS OBJETOS BIOTECNOLÓGICOS: CONCEPÇÕES FILOSÓFICAS E CONSEQÜÊNCIAS PARA SUA AVALIAÇÃO}

Resumo: Por sua própria natureza, os sistemas biotecnológicos geram tanto benefícios como riscos e situações de dúvidas e de ignorância. As avaliações dos sistemas biotecnológicos, dos artefatos que produzem e de suas conseqüências nas sociedades e na natureza, dependem tanto de valores constitutivos destes sistemas biotecnológicos, como de diferentes valores externos a eles, que variam de um grupo social a outro, e que muito dificilmente chegam a coincidir completamente. Tal diversidade valorativo deve ser reconhecida nos processos de avaliação biotecnológica, por exemplo sobre questões de biossegurança, por parte de experts e de diversos setores sociais (agentes estatais, empresários, organizações não governamentais, grupos de cidadãos). Consequentemente, devem estabelecer-se instâncias de participação de experts de diferentes, disciplinas naturais, sociais e humanisticas, bem como de não peritos, representantes de setores sociais com interesses em jogo, que vigiem o impacto dos sistemas biotecnológicos e de seus artefatos, e que tenham capacidade de decidir e de realizar ações para prevenir, ou enfrentar os efeitos negativos dos sistemas biotecnológicos.

Palavras chave: Biotecnologia, ética e biotecnologia, natureza dos objetos biotecnológicos, sistemas biotecnológicos, artefatos biotecnológicos.

* Investigador y Profesor de Filosofía en el Instituto de Investigaciones Filosóficas de la Universidad Nacional Autónoma de México. Correspondencia: olive@servidor.unam.mx 


\section{Introducción}

El desarrollo científico y tecnológico en el siglo XX, particularmente en su segunda mitad, estuvo marcado por el surgimiento y el crecimiento de los sistemas que muchos autores llaman "tecnocientíficos". Algunos ejemplos paradigmáticos de sistemas tecnocientíficos los encontramos en la investigación nuclear, en la espacial, en la informática y en el desarrollo de las redes telemáticas. Pero quizá entre los ejemplos que hoy en día más acaparan la atención pública, y atraen a los mayores intereses económicos y militares, se encuentran la investigación genómica, la ingeniería genética y la biotecnología en general.

En este artículo discutiremos una caracterización de los objetos biotecnológicos como un tipo particular de objetos tecnológicos. Veremos que conviene distinguir entre sistemas biotecnológicos y artefactos biotecnológicos, y que muchos sistemas y artefactos biotecnológicos son además tecnocientíficos.

No se trata sólo de proponer una nomenclatura aparentemente novedosa (y farragosa) para referirse a fenómenos y objetos bien conocidos. El problema es que en la segunda mitad del siglo XX surgieron disciplinas tecnocientíficas -entre ellas las biotecnológicasque actualmente están teniendo un gran impacto social (en lo económico y en lo cultural), así como ambiental, y es previsible que sus efectos aumenten en el futuro. Para comprender ese impacto y, en su caso, para tomar decisiones que permitan intervenir en la sociedad y en el ambiente, por ejemplo para paliar efectos negativos, para prevenirlos, o para remediarlos se requiere entender primero la naturaleza de esos objetos. Y puesto que se trata de objetos novedosos en la historia de la humanidad, que tendrán consecuencias en la sociedad y en el ambiente hasta ahora no conocidas, necesitamos nuevos conceptos para percibirlos, observarlos, entenderlos y poder actuar sobre ellos.

Una de las preguntas que nos interesa discutir es: ¿cómo situarse frente y cómo juzgar a la biotecnología? ¿Cómo situarse frente a los objetos biotecnológicos y cómo evaluar su uso y sus consecuencias? El problema axiológico, el problema de la evaluación de los fines y valores implicados, no puede abordarse adecuadamente si no se comprende la naturaleza de los objetos $\mathrm{y}$ fenómenos que se pretende evaluar.

\section{Técnicas, artefactos y sistemas tecnológicos}

Comencemos por recordar que no hay una única manera legítima de concebir a la ciencia, ni a la tecnología, ni a la tecnociencia. Muchas veces se entiende a la tecnología como reducida a un conjunto de técnicas o, en todo caso, de técnicas y de artefactos. Pero así entendida no es posible dar cuenta de ella y de su importancia en el mundo contemporáneo, y menos hacer propuestas sensatas para su evaluación. Una buena aproximación a la tecnología la ha ofrecido Miguel Ángel Quintanilla (1-3), quien ha aclarado la distinción entre técnicas, artefactos y sistemas tecnológicos. (Esta sección se apoya en la parte segunda de "El Bien, el Mal y la Razón"(4), donde se desarrollan más a fondo los conceptos que aquí se explican).

Las técnicas son sistemas de habilidades y reglas que sirven para resolver problemas. Las técnicas se inventan, se comunican, se aprenden y se aplican. Por ejemplo, podemos hablar de un grabado hecho con la técnica de "punta seca", de técnicas para resolver sistemas de ecuaciones, de técnicas de propaganda para ganar el mercado para un cierto producto o de técnicas para clonar organismos vivos.

Los artefactos son objetos concretos que se usan al aplicar técnicas y que suelen ser el resultado de las transformaciones de otros obje- 
tos concretos. Los artefactos se producen, se fabrican, se usan y se intercambian. Vivimos rodeados de artefactos: televisores, teléfonos, computadoras, aviones, organismos genéticamente modificados. También un organismo clonado, como Dolly, es un artefacto, porque es el resultado de un sistema de acciones intencionales humanas que han aplicado técnicas y, con base en un complejo de saberes científicos y tecnológicos, han transformado objetos concretos para generar un nuevo objeto: el artefacto (la criatura Dolly).

Pero la tecnología es mucho más que el conjunto de artefactos. Ni las técnicas ni los artefactos existen al margen de las personas que las aplican o los usan con determinadas intenciones. Una piedra bruta no ha sido fabricada por nadie, no es un artefacto, pero puede ser usada como instrumento para pulir otra piedra, para romper una nuez o una cabeza. Cuando alguien la usa intencionalmente para transformar un objeto concreto y producir así un artefacto, entonces se ha creado un sistema tecnológico. Éste es el principal concepto para entender y evaluar a la tecnología y sus impactos en la sociedad y en la naturaleza.

Un sistema tecnológico consta de agentes intencionales que persiguen al menos un fin. Digamos un grupo de científicos y de empresarios que buscan producir una nueva vacuna para luego comercializarla. El sistema incluye también a los objetos que los agentes usan con propósitos determinados (una piedra que se utiliza para pulir otra piedra y fabricar un cuchillo, o instrumentos utilizados para modificar genes y producir así organismos con determinadas características fenotípicas). Asimismo el sistema contiene al menos un objeto concreto que es transformado (la piedra que es pulida, los genes que son modificados). El resultado de la operación del sistema tecnológico, el objeto que ha sido transformado intencionalmente por alguien, es un artefacto (el cuchillo, o un organismo genéticamente modificado).

Los resultados de un sistema tecnológico pueden ser aparatos (aviones de combate o pulmones artificiales), sucesos (la muerte de personas, la destrucción o el empobrecimiento de la biodiversidad de una región o del planeta entero), o pueden ser procesos dentro de un sistema (la paulatina recuperación del estado de salud de una persona enferma o de un ecosistema en crisis, la constante reducción de la inflación en un sistema económico), o modificaciones de un sistema (las alteraciones en un sistema ecológico por la construcción de una presa, el cambio climático).

Al plantearse fines, los agentes intencionales de un sistema tecnológico lo hacen contra un trasfondo de creencias, de conocimientos y de valores. Alguien puede querer pulir una piedra porque cree que le servirá para cortar frutos. La piedra pulida es algo que el agente intencional considera valiosa. Un grupo de personas puede querer producir un medicamento o un órgano obtenido mediante técnicas de clonación, porque creen que el primero servirá para curar enfermedades o, el segundo, para restablecer el funcionamiento normal del organismo de una persona; en ambos casos se presupone que la salud de las personas es valiosa. Los sistemas tecnológicos, entonces, también involucran creencias y valores.

Las creencias se derivan de la capacidad de las personas de representarse conceptualmente la realidad sobre la cual desean intervenir. Los agentes intencionales que forman parte de los sistemas tecnológicos abstraen de la realidad ciertos aspectos que les interesan, construyen modelos y teorías para explicárselos y para intervenir sobre ellos, para modificarlos o para manipularlos. Los valores son asignados por los seres humanos a estados de cosas en el mundo cuando los consideran como buenos o malos, o como deseables o indeseables. 
Todo esto significa que los seres humanos son capaces de tomar decisiones y promover la realización de ciertos estados de cosas mediante el diseño y la aplicación de sistemas tecnológicos, en función de sus representaciones, creencias, conocimientos, intereses, valores, deseos y preferencias. Pero en la evaluación de un sistema tecnológico y de sus resultados pueden chocar sistemas de valores y de intereses, produciéndose juicios encontrados. Por ejemplo, los materiales de construcción que se obtienen mediante la explotación de un bosque, digamos la madera, pueden ser valiosos para un grupo humano. Pero si eso conduce a la deforestación de un valle, el proceso de tala puede ser juzgado indeseable por algunos grupos. La sustitución de cultivos tradicionales por otros con semillas modificadas genéticamente puede ser valiosa para ciertos sectores sociales, por ejemplo por razones económicas, pero indeseable para otros grupos, porque se afecta de manera negativa e irreversible la riqueza de la biodiversidad.

Hoy en día los sistemas tecnológicos pueden ser muy complejos. Pensemos tan sólo en una planta núcleo-eléctrica, en sistemas de salud preventiva en donde se utilizan vacunas o en un sistema agrícola donde se cultivan organismos genéticamente modificados. Estos sistemas, además de ser complejos de acciones, involucran conocimientos científicos entre muchos otros elementos (de física atómica en un caso y de biología en los otros). En estos sistemas está imbricada indisolublemente la ciencia y la tecnología, por eso suele llamárseles sistemas tecnocientificos (5, 6).

Por "sistemas tecnocientíficos" entenderemos, pues, sistemas tecnológicos que constan de un complejo de saberes, prácticas, sistemas de acciones e instituciones, en los que la ciencia y la tecnología son interdependientes. Para Javier Echeverría "la tecnociencia se caracteriza porque no hay avance científico sin avance tecnológico $\mathrm{y}$, recíprocamente, $[\ldots]$ cuando el conocimiento científico depende estrictamente de los avances tecnológicos, de modo que no es posible observar, medir ni experimentar sin recurrir a grandes equipamientos, entonces estamos hablando de tecnociencia"(6, p. 222).

Los sistemas tecnocientíficos, como los científicos, buscan describir, explicar o predecir lo que sucede, pero no se limitan a ello, también tienen, como la tecnología, el propósito central de intervenir en partes del mundo natural y social y de transformarlas. Aunque las tecnociencias han tenido un crecimiento espectacular en las tres últimas décadas y han desplazado en importancia económica y social a las ciencias y a las tecnologías tradicionales, éstas no han sido eliminadas. Lejos de ello, más bien asistimos hoy a una convivencia de técnicas, sistemas científicos, sistemas tecnológicos y sistemas tecnocientíficos.

\section{Sistemas biotecnológicos y artefactos biotecnológicos: lo natural y lo artificial}

Los artefactos son productos de sistemas de acciones intencionales, pero no todo artefacto es producido intencionalmente, ni sólo los aparatos son artefactos. Hay consecuencias de los sistemas tecnológicos que no son intencionales y por lo general no son previstas $\mathrm{y}$, sin embargo, son artificiales.

Los sucesos, los procesos o las modificaciones de los sistemas naturales o sociales son artificiales, tanto como los aparatos, cuando son efecto de la operación de un sistema tecnológico. La muerte de una persona puede ser natural, debida a una enfermedad que su cuerpo ya no puede superar, pero es (un suceso) artificial si resulta de la acción intencional de alguna persona (aunque la intención de quien actuó no haya sido producir la muerte de aquella persona, es decir, aun cuando esa muerte haya sido una consecuencia no buscada, ni deseada, ni 
prevista). La muerte de la Princesa Diana, como consecuencia de la persecución de los fotógrafos sensacionalistas, fue un suceso artificial, pero no fue buscado intencionalmente por nadie (o al menos eso suponemos). La destrucción de una ciudad por un terremoto es natural, pero es artificial si es causada por la explosión de una bomba nuclear.

Fernando Broncano menciona un bello ejemplo de un grupo de cazadores y recolectores que, cada día, después de la ardua jornada, regresan a su aldea. Su objetivo intencionalmente buscado es llegar a casa por el trayecto más sencillo. Con el tiempo, regresando por la misma ruta todos los días, el resultado es un sendero en el paisaje(7, p. 102). Broncano sostiene que la intencionalidad con la que se produce un cierto resultado es una condición necesaria pero no suficiente para distinguir lo natural de lo artificial. Para él, la característica esencial de lo artificial se encuentra en lo que denomina "composicionalidad de segundo orden, o capacidad para fabricar instrumentos que produzcan instrumentos", y se trata de una "característica específicamente humana" (7, p. 130). "Muchos animales disponen de técnicas, es decir, de patrones estables de conducta que transforman el medio, y son también muchos los animales que fabrican artefactos". Pero él prefiere en definitiva la idea de la composicionalidad de segundo orden, que toma del antropólogo Steven Mithen: "hay un salto cualitativo en la evolución cuando se comienzan a construir instrumentos para fabricar instrumentos"(7, p. 114).

Desde nuestro punto de vista, que un objeto sea producido intencionalmente (deliberadamente) no es una condición necesaria para ser un artefacto. La intención de los cazadores nunca fue construir el sendero. Su objetivo intencionalmente buscado era regresar a casa. El sendero no es un objeto producido intencionalmente, sin embargo es un artefacto, no es un producto sólo natural, pues no hubiera existido de no ser por las acciones intencionales de un grupo de seres humanos, aunque su fin deliberadamente buscado era otro. La intencionalidad es necesaria como componente del sistema (tecnológico) de acciones bajo el cual se busca obtener un cierto fin. Pero además de los fines buscados deliberadamente (que a veces no se logran, o no todos), el sistema de acciones puede generar otras consecuencias que muchas veces ni siquiera son previstas y, en ocasiones, tampoco son deseadas.

Broncano ha señalado, acertadamente, que el problema no consiste en encontrar una línea de demarcación tajante entre lo natural y lo artificial, sino en distinguir dentro de los objetos naturales aquéllos que además son artificiales(7, p. 101). Así, por ejemplo, la capa de ozono es un objeto natural, y el fenómeno que llamamos su adelgazamiento, o el objeto llamado "agujero de ozono", no deja de ser un objeto de la naturaleza. Ciertamente es un fenómeno que no fue buscado intencionalmente por nadie. Pero es un producto de una compleja cadena de relaciones causales, entre cuyos elementos se encuentran sistemas de acciones intencionales de seres humanos, que produjeron y utilizaron los CFC's para otros fines específicos (refrigeración, latas de aerosol, etc.). El adelgazamiento de la capa de ozono es, pues, una consecuencia de un sistema de acciones humanas intencionales, y por eso es un resultado artificial, es un artefacto, aunque no haya sido buscado, ni previsto, ni deseado. A continuación veremos la importancia de esta nota para la evaluación de los sistemas biotecnológicos.

\section{La noción de riesgo y la biotecnología}

Una de las características de los sistemas biotecnológicos, especialmente de los tecnocientíficos, es la siguiente: en virtud de su propia naturaleza, producen en su entorno -social y ambiental- efectos a corto, mediano y largo plazo, 
muchos de los cuales son significativos para los seres humanos aunque no hayan sido buscados. Una buena parte de esos efectos son imposibles de predecir en el momento de la puesta en funcionamiento del sistema tecnocientífico, por ejemplo cuando se libera al ambiente un organismo genéticamente modificado; algunos de ellos serán valorados posteriormente como positivos y otros como negativos y, generalmente, la valoración variará de unos grupos sociales a otros.

Esto significa que los sistemas biotecnológicos generan situaciones de riesgo, es decir, situaciones en las que se pone en juego algo valioso para un grupo de seres humanos a partir de posibles consecuencias de la acción o de la operación de un cierto sistema (natural o artificial). En tales situaciones puede darse alguno de estos tres casos: i) que se conozcan las probabilidades de la ocurrencia de cada uno de los resultados posibles; ii) que se desconozcan tales probabilidades, en cuyo caso la situación es de incertidumbre; o iii) que ni siquiera se sepa cuáles sucesos pueden ocurrir como consecuencia de la aplicación del sistema, entonces la situación es de ignorancia.

Las situaciones de riesgo tienen estas tres características:

1) Son situaciones de elección. El riesgo surge a partir de decisiones humanas de actuar y producir algo, o de omitir acciones y dejar que pase algo $(8, p .23)$.

2) Cuando ocurren daños, puesto que han sido posibles en virtud de decisiones humanas, las situaciones de riesgo implican la atribución de alguna responsabilidad.

3) Son situaciones que involucran problemas de justicia social pues, en las sociedades contemporáneas, "los conflictos sociales sobre riesgos pueden entenderse, por lo menos en parte, como conflictos respecto a la compensación por los riesgos, lo que necesariamente entraña también conflictos sobre el reparto de bienes" $(8, p .25)$.

Enfocando el riesgo de esta manera, es un corolario que su identificación, estimación, aceptabilidad y gestión -visto todo esto como un continuo y no como compartimentos estancos-, necesariamente dependen de valores(9). Aunque no todos los valores involucrados son de tipo ético(10), existe un problema ético de base en las formas de enfrentar los problemas del riesgo que generan los sistemas biotecnológicos. Pues la información y el conocimiento pertinentes para la identificación, estimación y gestión del riesgo siempre dependen de un contexto, de la posición de quienes evalúan, de sus fines, intereses y valores; y "tomar una posición, y estar en una posición es, inevitablemente, una cuestión de ética" $(11, p .4)$.

Las percepciones del riesgo están íntimamente ligadas a la forma como los seres humanos, desde diferentes posiciones, comprenden los posibles fenómenos que constituyen peligros o amenazas. No hay una única comprensión correcta del riesgo en cada situación específica, como tampoco hay una única y correcta manera de estimarlo y gestionarlo. Esta es la idea que se ha ocultado mediante la tradicional asociación del "lenguaje del riesgo" con el mundo de la economía, del comercio, de la medicina profesional, de los deportes peligrosos y de los seguros. Este punto de vista sostiene engañosamente que "la percepción del riego implica una relación particular con un futuro desconocido cuya posibilidad, de llegar a realizarse, podría sin embargo calcularse mediante extrapolaciones de ocurrencias pasadas"(11, p. 7). Desde esa concepción, la evaluación del riesgo se reduce a una mera cuestión matemática que debe quedar en manos sólo de expertos.

Esta interpretación del riesgo cumple una función ideológica al ocultar que las situaciones 
de riesgo, por su propia estructura, admiten una pluralidad de puntos de vista distintos - que pueden ser todos correctos-al percibir, identificar, evaluar y gestionar el riesgo. Esto es así, entre otras razones, porque hay sistemas de valores que son constitutivos de esas situaciones, y porque la comprensión y evaluación de una situación de riesgo necesariamente se basa en valores, y los sistemas de valores varían en función de quienes hacen la evaluación.

Cuando se trata de evaluar los riesgos de la aplicación de sistemas biotecnológicos, por ejemplo, de la liberación de un organismo genéticamente modificado al ambiente, no hay un acceso privilegiado a la verdad, a la objetividad o a la certeza del conocimiento y, por eso, en estas tareas deben participar al mismo nivel los científicos naturales, los científicos sociales, los tecnólogos, los humanistas, los trabajadores de la comunicación, los empresarios, los políticos, los ciudadanos y todas las personas cuyas vidas pueden ser afectadas. Esto no significa desconocer que diferentes sectores de la sociedad, y diferentes miembros dentro de esos distintos sectores, tienen un acceso diferenciado a la información pertinente, al saber especializado, y a ciertos recursos necesarios para conocer y evaluar las consecuencias de la biotecnología. Pero sí quiere decir que, cuando se trata de evaluar resultados y decidir acciones en torno a un sistema biotecnológico que afecta a la sociedad o al ambiente, la visión y las conclusiones de cada sector serán necesariamente incompletas, y ninguno tiene un privilegio que justifique su participación a costa de excluir a otros sectores que pueden aportar otros puntos de vista valiosos y pertinentes.

\section{Consecuencias para la bioética y políticas públicas en torno a problemas planteados por la biotecnología}

¿Cómo situarse frente y cómo evaluar a los sistemas biotecnológicos y a los artefactos que producen, así como a las consecuencias que generan, cuando se afectan intereses colectivos de diversos sectores de la sociedad? ¿Cómo contender con estos problemas en las sociedades contemporáneas que aspiran a vivir bajo una organización democrática?

Las sociedades modernas se basan en un modelo de ciudadano que proviene de uno de los supuestos mejor atrincherados del pensamiento moderno, a saber, que las personas son racionales y autónomas. Por otra parte, las modernas sociedades democráticas se caracterizan por la convivencia de muy diversos grupos y sectores sociales, con diferentes visiones del mundo y diversos sistemas de valores. $\mathrm{La}$ identificación, evaluación y propuestas de gestión del riesgo dependen de esos sistemas de valores $\mathrm{y}$, puesto que ninguno de ellos está por encima de los demás, entonces no hay una única manera correcta de identificar los riesgos, ni una única estimación acertada, ni una valoración que sea la única justa. Por lo tanto, tampoco es posible una visión sobre la gestión del riesgo que sea la única correcta y éticamente aceptable; puede haber diferentes puntos de vista tan legítimos unos como otros. No se trata de una visión relativista que sostenga que cualquier punto de vista es tan bueno como cualquier otro. Se trata más bien de una concepción pluralista que sostiene que no existe un punto de vista que sea el único correcto.

Esta situación de pluralidad, que se da tanto para los problemas del conocimiento en general como para cuestiones éticas (en particular, para los problemas de la identificación, evaluación y gestión del riesgo), exige que, para encontrar soluciones justas, la toma de decisiones debe ser resultado de un amplio proceso dialógico. En tal proceso, las diversas partes interesadas deben intercambiar información, proponer y rebatir con razones los métodos que se deben seguir. Deben ventilar y discutir abiertamente los intereses, fines y valores de todos los sectores sociales involucrados y afectados. 
Finalmente, deben debatir las formas propuestas para evaluar y gestionar los riesgos en cuestión, así como para intentar prevenir, atenuar o compensar los daños, buscando siempre alcanzar acuerdos aceptables para las diversas partes.

Para lograr lo anterior, se requiere un conjunto de "normas éticamente justificables" que animen y regulen la participación pública en el proceso de identificación, evaluación y gestión del riesgo generado por los sistemas biotecnológicos. En el contexto plural de las sociedades modernas, la estabilidad de los acuerdos exige que tales normas sean consideradas como legítimas por los diversos grupos sociales. Esto se logrará sólo cuando los miembros de cada uno de los grupos significativos acepten esas normas por razones que ellos consideren válidas, aunque tales razones no sean las mismas para todos los sectores sociales ni para todos los ciudadanos, pues dependerán de sus particulares visiones del mundo, de sus diversos sistemas de valores y de sus principios morales y religiosos.

Por ejemplo, ésta es la situación típica que se plantea en los Estados democráticos laicos con respecto a la legislación sobre el aborto. Puesto que un Estado laico no debe comprometerse con ningún punto de vista moral particular en torno a la admisibilidad o condena moral del aborto, debe llegar a un acuerdo con sus ciudadanos acerca de las normas que regularán las decisiones y acciones del Estado al respecto. Tales normas deberían permitir que los ciudadanos actúen de acuerdo con sus particulares valores y principios morales y no deberían, por ejemplo, obligar al Estado a imponer un castigo a las mujeres, o a las parejas, que decidan un aborto, pues la condena del aborto depende de valores y principios morales específicos que varían de un grupo social a otro, y en relación con los cuales el Estado laico no debe pronunciarse. Esas normas que regulen la actuación del Estado deberían ser acep- tables para todos los ciudadanos -incluyendo aquellos que condenan moralmente el abortocon base en una actitud tolerante con otros puntos de vista morales, actitud necesaria para lograr una convivencia armoniosa entre diversos grupos sociales, religiosos o étnicos, en el marco del Estado laico.

Podemos resumir de la siguiente manera las razones para justificar la participación pública en el diseño, evaluación y gestión de políticas en materia de biotecnología, así como en la identificación, estimación y gestión del riesgo generado por los sistemas biotecnológicos:

i) Si se niega la participación pública, y las decisiones en estas cuestiones se dejan sólo en manos de los expertos, entonces se genera una "tecnocracia" - es decir un sistema donde las decisiones que afectan a todos los ciudadanos y al ambiente son tomadas sólo por pequeños grupos de especialistas-, solución que resulta incompatible con los valores democráticos de equidad en la pluralidad de los puntos de vista, el derecho a la decisión libre de todos, y la igualdad de todos en la decisión del gobierno (12, p. 336).

ii) El desarrollo de la biotecnología afecta en tal grado a la naturaleza y a la sociedad, que el diseño, la evaluación y la gestión de políticas y de riesgos en materia de biotecnología implican decisiones sobre restricciones de posibles cursos de investigación y de posibles aplicaciones porque podrían ser perniciosas. En la evaluación del impacto de los sistemas biotecnológicos están involucrados problemas de distribución de bienes y beneficios, atribución de responsabilidades y sanciones, así como de exigencia de compensaciones. Es decir, se trata de dirimir cuestiones incluso de justicia social, cuya resolución en una sociedad democrática resultaría ilegítima sin una amplia participación pública. 
iii) Si bien todas las sociedades requieren de expertos para resolver muchos de sus problemas, entre ellos la generación de medios para satisfacer las necesidades básicas de los ciudadanos - es decir, las necesidades indispensables para la realización de cualquier plan de vida-, y eso justifica la asignación de recursos sociales a esos grupos de expertos, en las sociedades democráticas es éticamente justificable exigir, primero, que los dineros públicos se asignen con el convencimiento y aprobación del público y, segundo, que los sistemas de ciencia y tecnología respondan a genuinas demandas de los ciudadanos que los mantienen, es decir, es un deber que esos sistemas tengan resultados que satisfagan genuinas necesidades sociales. Pero la identificación de las genuinas demandas sociales, incluyendo la determinación de las necesidades básicas de los ciudadanos, sólo puede ser legítima si surge de una amplia participación ciudadana, y no de la manipulación de grupos de interés y de poder.

Mencionemos, finalmente, sólo un par de ejemplos de mecanismos de participación ciudadana que es posible desarrollar. 1) Coloquios de consenso. Reuniones públicas que permiten a grupos de ciudadanos participar en la evaluación de sistemas tecnológicos específicos. Se trata de un diálogo entre ciudadanos y expertos, abierto al público y a los medios de comunicación. Por ejemplo en Dinamarca, a partir de este tipo de reuniones, se han tomado decisiones para prohibir ciertas tecnologías de preservación de alimentos o para prohibir a las empresas que exijan un perfil de salud de ADN a sus empleados y a quienes soliciten empleo.

2) Talleres de discusión de escenarios. Reuniones locales para propiciar el diálogo entre cuatro grupos de agentes: a) responsables de la elaboración de políticas; b) representantes de empresarios; c) expertos; d) grupos de ciuda- danos. En Dinamarca se ha utilizado para discutir temas como "ecología urbana" o "la biblioteca del futuro"(13).

\section{Conclusiones}

1) El desarrollo de la biotecnología y la aplicación de sus resultados, como la globalización, es un proceso que nadie puede detener, ni conviene intentarlo. Pero los seres humanos, en los diferentes papeles sociales que desempeñan (científicos, tecnólogos, políticos, gobernantes, legisladores, administradores públicos, funcionarios de organizaciones internacionales, empresarios y los ciudadanos de la calle), pueden tomar medidas y promover acciones que podrían influir en el desarrollo de los sistemas biotecnológicos y, sobre todo, encauzar sus beneficios y su impacto en la sociedad y en el planeta.

2) Es absurdo hacer juicios generales tales como: "la ciencia es buena" o "la ciencia es mala", "la tecnología es buena" o "la tecnología es mala", "la biotecnología es buena" o "la biotecnología es mala", "los organismos genéticamente modificados son buenos" o "los organismos genéticamente modificados son malos". Las evaluaciones deben hacerse siempre sobre sistemas tecnocientíficos específicos y sobre sus resultados y consecuencias (intencionalmente buscados o no).

3) Los sistemas y artefactos biotecnológicos y sus aplicaciones llevan consigo riesgos. Por esto, deben establecerse mecanismos de identificación, evaluación y gestión del riesgo que generan.

4) Se debe reconocer que en torno a la biotecnología y sus aplicaciones se congregan y enfrentan intereses económicos, militares, sociales, culturales y ambientales, que muchas veces son incompatibles. 
5) Dado que los sistemas biotecnológicos generan incertidumbre e ignorancia, y en virtud de que existe una amplia diversidad de valores y de intereses en juego, ya no es aceptable en las sociedades democráticas que las decisiones se tomen sólo con base en la opinión de expertos. Para la toma de decisiones se requiere la participación de muy diversos grupos de expertos y de no expertos.

6) En el campo legislativo y jurídico debe propiciarse el establecimiento de los debidos mecanismos de vigilancia y control de los posibles efectos de los sistemas biotecnológicos que permitan: a) tomar decisiones en cuanto a restricciones sobre cursos de investigación y sobre posibles aplicaciones de sistemas biotecnológicos específicos, porque podrían ser perniciosos; b) tomar decisiones sobre cómo determinar cuándo ciertas investigaciones o aplicaciones podrían ser perniciosas; c) tomar decisiones sobre formas de dirimir disputas y, en su caso, para fincar responsabilidades, sobre todo cuando se dañen bienes públicos (como el ambiente o un entorno cultural); d) tomar decisiones para exigir compensaciones. Dichos mecanismos deben desarrollarse a niveles locales, nacionales, regionales e internacionales.

7) ¿Quiénes deberían participar en esas instancias y en esas discusiones? La legislación no puede prever de antemano todos los agentes interesados y pertinentes en relación con cada problema específico. Por lo tanto, debe prever los mecanismos que garanticen en cada caso la participación de diferentes grupos de expertos y de diferentes sectores sociales cuyos intereses van en juego.

8) La obligación de los estados y de los organismos internacionales es evitar moratorias o prohibiciones generales absurdas -como moratorias generales sobre investigaciones biotecnológicas-y, en cambio, cuando haya razones públicamente debatidas y generalmente aceptadas, deben establecer moratorias específicas o prohibiciones concretas como, por ejemplo, sobre clonación humana con fines reproductivos.

9) Los expertos tienen las siguientes obligaciones: a) ser transparentes en cuanto a lo que saben y en cuanto a lo que ignoran; b) hacer públicas las razones que respaldan sus opiniones; c) nunca subestimar o despreciar a la gente (ni al ciudadano de la calle ni al legislador); d) reconocer que su papel como expertos tiene un límite.

10) Los problemas que plantea la biotecnología ya no pueden resolverse sólo con "más ciencia" o sólo con más expertos (aunque en general sea conveniente investigar más, e incrementar el número de especialistas). No existe ya un único grupo ni un conjunto definido de grupos de expertos que pueda tomar las decisiones importantes (por ejemplo, sobre atribución de responsabilidades, determinación de compensaciones o sobre medidas de seguridad). Se requieren también novedosas formas de organización social que estimulen mayor participación ciudadana, lo cual supone mejor educación, así como un "nuevo contrato social sobre la ciencia y la tecnología"(14).

11) Según el "nuevo contrato social sobre la ciencia y la tecnología", a la sociedad le conviene apoyar el fortalecimiento y desarrollo de núcleos de expertos que generen conocimiento fiable y útil para resolver sus problemas. La biotecnología, sin duda, constituye uno de los instrumentos de mayor fiabilidad para resolver muchos de esos problemas. Pero las comunidades de expertos, por su parte, deben reconocer que, en virtud de los riesgos que generan los sistemas bio- 
tecnológicos, la vigilancia y la propuesta de soluciones a problemas específicos, como los que pueden derivarse de la aplicación de sistemas biotecnológicos, deben ser tomadas por medio de mecanismos que aseguren tanto la participación ciudadana como la de los grupos de expertos pertinentes. Por otra parte, puesto que los recursos que permiten el desarrollo de la biotecnología, sean públicos o privados, provienen del trabajo de los ciudadanos, los sistemas biotecnológicos deberían abocarse a la resolución de problemas planteados por los diversos sec- tores sociales, y no responder únicamente a los intereses de los sectores empresariales o militares.

12) La tecnología en general, y la biotecnología en particular, cambia las formas de vida de la gente. La decisión de aceptar o no los cambios en su forma de vida corresponde a la gente, no a los expertos, ni al estado. Por eso debe haber discusión pública acerca de cuáles cambios en la forma de vida, inducidos por los sistemas biotecnológicos, son deseables y éticamente aceptables.

\section{Referencias}

1. Quintanilla MA. Tecnología: un enfoque filosófico. Madrid: Fundesco; 1989.

2. Quintanilla MA. Educación moral y tecnológica. En: Olivé L, Villoro L, eds. Educación Moral e Historia. Homenaje a Fernando Salmerón. México: UNAM; 1996: 315-332.

3. Quintanilla MA. Técnica y cultura. Teorema 1998; XVII (3): 49-69.

4. Olivé L. El Bien, el Mal y la Razón. Facetas de la ciencia y la tecnología. México: Paidós; 2000.

5. Echeverría J. Filosofia de la Ciencia. Madrid: Ediciones Akal; 1995.

6. Echeverría J. Tecnociencia y sistemas de valores. En: López Cerezo JA, Sánchez Ron JM, eds. Ciencia, Tecnología, Sociedad y Cultura. Madrid: Biblioteca Nueva-OEI; 2001: 221-230.

7. Broncano F. Mundos Artificiales, Filosofia del cambio tecnológico. México: Paidós; 2000.

8. López Cerezo JA, Luján JL. Ciencia y Política del Riesgo. Madrid: Alianza Editorial; 2000.

9. Jaeger CC, Renn O, Rosa E, Webler Th. Risk, Uncertainty and Rational Action. London: Earthscan Publications Ltd; 2001.

10. Echeverría J. Ciencia y Valores. Barcelona: Destino; 2002.

11. Adam B, Beck U, Van Loo J. The Risk Society and Beyond. Critical issues for social theory. London: Sage Publications Ltd; 2000.

12. Villoro L. El Poder y el Valor. México: Fondo de Cultura Económica; 1997.

13. Andersen IA, Jaeger B. Scenario workshops and consensus conferences: towards more democratic decision-making. Science and Public Policy 1999, 26(5):331-340.

14. Consejo Internacional para la Ciencia (ICSU). La Ciencia para el siglo XXI, Un nuevo compromiso: Declaración sobre la ciencia y el uso del saber científico. París: UNESCO; 2000. 